\title{
Nivolumab plus ipilimumab in non-small-cell lung cancer
}

\author{
Martin Reck*,1, Hossein Borghaei ${ }^{2}$ \& Kenneth J O'Byrne ${ }^{3}$ \\ ${ }^{1}$ LungenClinic Grosshansdorf, Airway Research Center North, German Center for Lung Research, Woehrendamm 80, 22927 \\ Grosshansdorf, Germany \\ ${ }^{2}$ Division of Thoracic Medical Oncology, Department of Hematology/Oncology, Fox Chase Cancer Center, 333 Cottman Avenue, \\ Philadelphia, PA 19111, USA \\ ${ }^{3}$ Princess Alexandra Hospital, Translational Research Institute and Queensland University of Technology, Brisbane, QLD 4102 , \\ Australia \\ *Author for correspondence: Tel.: +49 4102601 2101; Fax: +49 4102601 7101; m.reck@lungenclinic.de
}

Nivolumab and ipilimumab, two therapeutic immune checkpoint inhibitor antibodies that block PD-1 and CTLA-4, respectively, have indications in cancer as single agents and in combination. In this Review, we examine the potential role of dual immune checkpoint inhibition with nivolumab plus ipilimumab in the management of patients with previously untreated advanced non-small-cell lung cancer, based on results from the Phase III CheckMate 227 study. Immunotherapies with indications in the first-line treatment of non-small-cell lung cancer include pembrolizumab alone and combined with chemotherapy, and atezolizumab combined with bevacizumab and chemotherapy. CheckMate 227 is the first Phase III study evaluating first-line chemotherapy-sparing combination immunotherapy and including tumor mutational burden as a biomarker for patient selection.

First draft submitted: 16 January 2019; Accepted for publication: 11 April 2019; Published online: 8 May 2019

Keywords: atezolizumab • CTLA-4 • immunotherapy • ipilimumab • nivolumab • non-small-cell lung cancer • pembrolizumab • PD-1 • PD-L1 • tumor mutational burden

Lung cancer is the most commonly diagnosed cancer and the leading cause of death from cancer worldwide, accounting for an estimated 18.4\% of all cancer-related deaths in 2018 [1]. In the USA, lung cancer has the second highest age-adjusted incidence among primary cancer types (after breast cancer) and is associated with by far the highest cancer-related mortality [2]. Global, age-standardized incidence rates for lung cancer are lower among women than men across all regions; however, high rates among women are seen in North America, Northern and Western Europe, and Australia/New Zealand [1]. Of an estimated 222,500 new cases of lung cancer in the USA in 2017, almost half (47\%) were diagnosed in women [3]. Non-small-cell lung cancer (NSCLC) accounts for 80-85\% of lung cancer cases in the USA [4]. Although smoking is the most common cause of NSCLC, approximately $10-15 \%$ of patients with NSCLC have never smoked [5].

Until a few years ago, the treatment for patients with advanced NSCLC with no known targetable driver mutations was essentially limited to systemic cytotoxic therapy, with platinum doublet chemotherapy as the standard first-line treatment. In randomized controlled trials, platinum-based therapies were associated with a median overall survival (OS) of 10-13 months and 2-year survival rates of 17-24\% from the time of treatment initiation [6-11]. Patients who experienced disease progression during first-line therapy had few remaining treatment options, including docetaxel, erlotinib, gefitinib or pemetrexed for nonsquamous NSCLC and docetaxel, erlotinib or gefitinib for squamous NSCLC. The recent introduction of immune checkpoint inhibitors [12] has greatly improved outcomes in many cancers, including NSCLC. Therapeutic antibodies that disrupt the interaction between PD-1 and PD-L1, in other words, PD-1 and PD-L1 inhibitors, are now the preferred second-line treatment option after first-line chemotherapy [13,14]. Furthermore, the PD-1 inhibitor pembrolizumab has been approved as monotherapy for the first-line treatment of patients with advanced NSCLC and high PD-L1 expression (tumor proportional score $\geq 50 \%$ ) $[15,16]$. To provide effective first-line treatment options for a broader range of patients, various combinations of PD-1 and PD-L1 inhibitors with chemotherapy versus chemotherapy alone are currently being evaluated in

Future Medicine 
randomized controlled trials. Recently, positive results reported for several of these studies [17-19] led to the marketing approval of pembrolizumab in combination with chemotherapy for the first-line treatment of patients with metastatic NSCLC $[15,16]$ and of the PD-L1 inhibitor atezolizumab in combination with bevacizumab and chemotherapy for the first-line treatment of patients with metastatic nonsquamous NSCLC [20]. As an alternative approach to combining immunotherapy and chemotherapy, dual immune checkpoint inhibition with nivolumab, a PD-1 inhibitor, and ipilimumab, an antibody that blocks CTLA-4, is being evaluated in the Phase III CheckMate 227 study.

In this Review, we will provide an overview of the current role of checkpoint inhibitors in the treatment of NSCLC, followed by an in-depth discussion of the available data on the efficacy and safety of nivolumab plus ipilimumab in patients with previously untreated advanced NSCLC. Particular emphasis will be given to the role of PD-L1 expression and tumor mutational burden (TMB) as biomarkers in the selection of patients for specific therapies. Of note, a discussion of the efficacy and safety of targeted therapy in patients with driver mutations sensitive to targeted therapy, particularly EGFR mutations and $A L K$ translocations, is not part of this Review and can be found elsewhere [21]. Similarly, emerging questions regarding the sequencing of different immunotherapies in the course of treating advanced NSCLC are not discussed in this Review.

\section{Market overview of immunotherapy for advanced NSCLC}

Second-line treatment: after chemotherapy

In randomized controlled studies, single-agent PD-1 or PD-L1 inhibitors, including nivolumab [22,23], pembrolizumab [24] and atezolizumab [25], were shown to significantly prolong OS compared with docetaxel in patients with previously treated advanced NSCLC. Based on these findings, current clinical practice guidelines recommend single-agent PD-1/PD-L1 inhibitors as the standard of care for patients with an ECOG performance status of 0 or 1 who progressed on or after first-line platinum-doublet chemotherapy and have no targetable $E G F R$ mutations or $A L K$ translocations [13,14]. Findings from an early Phase I trial of pembrolizumab showed that tumor PD-L1 expression affected treatment outcomes in both treatment-naive and previously treated patients [26], and patients with less than 1\% PD-L1 expression were excluded from the subsequent pivotal Phase II/III study of pembrolizumab for second-line treatment of advanced NSCLC [24]. However, results from pivotal Phase III studies of other PD-1 or PD-L1 inhibitors suggested that the relative treatment benefit of these therapies versus docetaxel may extend to patients with less than $1 \%$ tumor PD-L1 expression [22,23,25], warranting the inclusion of this patient population in the marketing approval of nivolumab and atezolizumab for the second-line treatment of advanced NSCLC [20,27-29].

A key advantage of immunotherapy over the limited chemotherapy options available after platinum-based chemotherapy is the greater durability of treatment benefit, as illustrated by the long-term survival data from multiple studies [30-33], including the Phase III CheckMate $017(\mathrm{~N}=272)$ and CheckMate $057(\mathrm{~N}=582)$ studies of nivolumab versus docetaxel in patients with previously treated squamous and nonsquamous advanced NSCLC, respectively. The estimated 2-year OS rates with nivolumab versus docetaxel were 23 versus $8 \%$ for patients with squamous NSCLC and 29 versus $16 \%$ for those with nonsquamous NSCLC [32]; the estimated 3-year OS rate for the combined CheckMate 017 and 057 study populations was 17\% with nivolumab versus $8 \%$ with docetaxel [33]. Further support for the durability of nivolumab benefit was provided by 5 -year follow-up data from a Phase I study that included 129 patients with previously treated advanced NSCLC who received nivolumab for up to 2 years [34]. The estimated 5-year survival rate was 16\% from the time of nivolumab treatment initiation. Of note, 12 of the 16 patients who survived at least 5 years received no subsequent therapy and were without evidence of progressive disease at last follow-up [34].

\section{First-line treatment}

Phase III studies evaluating the efficacy of single-agent PD-1/PD-L1 inhibitors as first-line therapy in advanced NSCLC sought to optimize outcomes by using PD-L1 expression level as a criterion for patient eligibility and/or stratification during randomization. In the landmark KEYNOTE-024 study, pembrolizumab significantly prolonged progression-free survival (PFS; the primary end point) and OS compared with platinum-doublet chemotherapy in patients whose tumors had $\geq 50 \%$ PD-L1 expression (tumor proportional score $\geq 50 \%$ ) [35], leading to its marketing approval for this patient population [15,16]. Median PFS was 10.3 months with pembrolizumab versus 6.0 months with chemotherapy [35], and median OS was 30.0 months with pembrolizumab versus 14.2 months with chemotherapy [36]. Recent results from the Phase III KEYNOTE-042 study in patients with $\geq 1 \%$ PD-L1 expres- 
sion further showed a significant OS advantage with pembrolizumab versus chemotherapy at a median follow-up of 12.8 months. The median OS was 16.7 months with pembrolizumab and 12.1 months with chemotherapy (hazard ratio [HR] for death: $0.81 ; 95 \% \mathrm{CI}: 0.71-0.93 ; \mathrm{p}=0.0018$ ). The OS advantage with pembrolizumab versus chemotherapy in KEYNOTE-042 was also significant in patients with $\geq 50 \%$ PD-L1 expression (HR: 0.69; 95\% CI: 0.56-0.85; p < 0.001), but results of an exploratory analysis suggested similar OS with pembrolizumab and chemotherapy in patients with 1-49\% PD-L1 expression (HR: 0.92; 95\% CI: 0.77-1.11) [37]. In contrast, results of CheckMate 026 showed no improvement of PFS (the primary end point) or OS with nivolumab versus chemotherapy in patients with $\geq 5 \%$ PD-L1 expression [38]. Although the reasons for the different results of these trials remain controversial and are likely multifactorial, the findings of CheckMate 026 highlighted the need for additional predictive biomarkers to identify patients who are most likely to respond to immunotherapy. Previous findings suggested that TMB as the principal driver of neoantigen burden is a critical determinant of the sensitivity of tumors to PD-1 inhibition in NSCLC [39]. Consistent with these findings, results of an exploratory post hoc analysis of CheckMate 026 showed improved PFS with nivolumab versus chemotherapy (median: 9.7 vs 5.8 months; HR for disease progression or death: 0.62 ; 95\% CI: 0.38-1.00) in patients with a high TMB, defined as $\geq 243$ somatic missense mutations per whole exome [38].

Since the approved indication of pembrolizumab monotherapy in the first-line setting is limited to patients with $\geq 50 \%$ PD-L1 expression in most countries, only an estimated $25-30 \%$ of all treatment-naive patients with advanced NSCLC who are tested for PD-L1 expression are eligible for this treatment [35]. To broaden the patient population that may benefit from first-line immunotherapy, various combination strategies are being pursued, including combinations of a PD-1 or PD-L1 inhibitor with histology-based chemotherapy and combination immunotherapy with nivolumab plus ipilimumab in TMB-selected and PD-L1-selected patient populations. Recent results from a number of Phase III studies underscore the overall success of these strategies.

KEYNOTE-189 and KEYNOTE-407 are ongoing randomized controlled Phase III studies evaluating the efficacy and safety of pembrolizumab plus chemotherapy versus chemotherapy alone in patients with advanced nonsquamous and squamous NSCLC, respectively. The results of interim analyses from both studies were recently published $[17,18]$. Both studies designated OS and PFS as the primary end points and used PD-L1 expression ( $\geq 1$ vs $<1 \%$ ) as a stratification factor during randomization. In KEYNOTE-189, median PFS was 8.8 months with pembrolizumab plus chemotherapy and 4.9 months with chemotherapy alone (HR for disease progression or death: 0.52; 95\% CI: 0.43-0.64; p < 0.001) [17]. Although median OS for the combination therapy was not reached at the median follow-up of 10.5 months, the available data showed a significant OS advantage with pembrolizumab plus chemotherapy versus chemotherapy alone (HR: 0.49; 95\% CI: 0.38-0.64; $\mathrm{p}<0.001$ ) and this survival advantage was preserved in all tested subgroups defined by PD-L1 expression level, including patients with less than 1\% PD-L1 expression [17]. In KEYNOTE-407, the median PFS was 6.4 months with pembrolizumab plus chemotherapy and 4.8 months with chemotherapy alone (HR for disease progression or death: 0.56 ; $95 \% \mathrm{CI}$ : $0.45-0.70 ; \mathrm{p}<0.001)$ [18]. The median OS was 15.9 months with pembrolizumab plus chemotherapy and 11.3 months with chemotherapy alone (HR for death: 0.64; 95\% CI: 0.49-0.85; $\mathrm{p}<0.001$ ). Subgroup analyses further suggested that the OS benefit of the combination was similar in patients with less than 1\% PD-L1 expression (HR for death: 0.61; 95\% CI: 0.38-0.98) and those with $\geq 1 \%$ PD-L1 expression (HR for death: 0.65 ; 95\% CI: $0.45-$ 0.92) [18]. Based on the findings of KEYNOTE-189 and KEYNOTE-407, the US FDA approved pembrolizumab in combination with pemetrexed and platinum chemotherapy for the first-line treatment of patients with metastatic nonsquamous NSCLC with no EGFR or $A L K$ aberrations, and pembrolizumab in combination with carboplatin and either paclitaxel or nab-paclitaxel for the first-line treatment of patients with metastatic squamous NSCLC [15]. A similar indication was granted by the EMA [16].

Several Phase III studies are evaluating combinations of atezolizumab and chemotherapy for the first-line treatment of advanced nonsquamous or squamous NSCLC. In the global Phase III IMpower150 study, the addition of atezolizumab to bevacizumab plus chemotherapy was evaluated in chemotherapy-naive patients with nonsquamous NSCLC, including patients with EGFR or $A L K$ alterations who were previously treated with at least one approved tyrosine kinase inhibitor [19]. Addition of atezolizumab to bevacizumab plus chemotherapy resulted in significantly longer PFS (HR: 0.62; 95\% CI: 0.52-0.74; p < 0.001) in patients without EGFR or $A L K$ alterations (irrespective of PD-L1 expression level) and patients with $E G F R$ or $A L K$ alterations previously treated with at least one approved tyrosine kinase inhibitor (HR: 0.59; 95\% CI: 0.37-0.94). Results of an interim OS analysis at a minimum follow-up of 14 months showed that median OS among patients without EGFR or $A L K$ alterations was 19.2 months with and 14.7 months without the addition of atezolizumab (HR: 0.78; 95\% CI: 
0.64-0.96; $\mathrm{p}=0.02$ ) [19]. In December 2018, the FDA approved atezolizumab in combination with bevacizumab, paclitaxel and carboplatin for the first-line treatment of patients with metastatic nonsquamous NSCLC with no $E G F R$ or $A L K$ genomic tumor aberrations [20]. In March 2019, the European Commission approved the use of this combination for the first-line treatment of metastatic nonsquamous NSCLC, with the additional provision that in patients with NSCLC with EGFR or $A L K$ genomic tumor aberrations, the combination is indicated only after failure of appropriate targeted therapies [40].

Results of IMpower130 presented at the ESMO 2018 Congress showed that the study met its coprimary end points of longer OS and PFS with atezolizumab plus chemotherapy (consisting of carboplatin and nab-paclitaxel) versus chemotherapy alone in the first-line treatment of patients with stage IV nonsquamous NSCLC with no $E G F R$ or $A L K$ alterations. Addition of atezolizumab to chemotherapy increased the median OS from 13.9 to 18.6 months (HR: 0.79; 95\% CI: 0.64-0.98; $\mathrm{p}=0.033$ ) and the median PFS from 5.5 to 7.0 months (HR: 0.64; 95\% CI: $0.54-0.77 ; \mathrm{p}<0.001$ ) [41]. Results of IMpower 132 demonstrated that addition of atezolizumab to carboplatin or cisplatin plus pemetrexed as first-line therapy significantly prolonged PFS (HR: 0.60; 95\% CI: 0.49-0.72; $\mathrm{p}<0.001)$ in patients with stage IV nonsquamous NSCLC. However, the results of an interim analysis of OS at a median follow-up of 14.8 months did not show a significant difference in OS between the two treatment arms (HR: 0.81; 95\% CI: 0.64-1.02; $\mathrm{p}=0.08$ ) [42].

Interim results from IMpower131, a large randomized Phase III study in patients with advanced squamous NSCLC, showed that PFS was longer with atezolizumab plus chemotherapy (carboplatin plus nab-paclitaxel) versus chemotherapy alone (6.5 vs 5.6 months; HR: 0.74; 95\% CI: 0.62-0.87); however, at a minimum follow-up of 12.8 months, no significant differences in OS were observed (HR: 0.92; 95\% CI: 0.76-1.12; $\mathrm{p}=0.41$ ) [43].

CheckMate 227 is evaluating the safety and efficacy of nivolumab-based therapies, including the combination of nivolumab plus chemotherapy, in biomarker-selected and unselected populations of patients with advanced NSCLC. PFS with nivolumab plus chemotherapy versus chemotherapy alone in patients with less than 1\% PD-L1 expression is a secondary end point in part 1 of the study. At a minimum follow-up of 11.2 months, median PFS was 5.6 months with nivolumab plus chemotherapy and 4.7 months with chemotherapy alone (HR: 0.74; 95\% CI: 0.58-0.94) [44]. Nivolumab plus chemotherapy as first-line therapy is being evaluated in unselected patients with advanced NSCLC in part 2 of Checkmate 227, with results expected in 2019.

Currently, pembrolizumab monotherapy for patients with $\geq 50 \%$ PD-L1 expression is the only chemotherapysparing therapy approved for the first-line treatment of advanced NSCLC. Combination immunotherapy with a PD-1/L1 inhibitor and a CTLA-4 inhibitor in patients selected by TMB, which has no meaningful correlation with PD-L1 expression $[38,45,46]$, has the potential to provide a chemotherapy-sparing treatment option for some patients who have less than 50\% PD-L1 expression.

As discussed in detail in a subsequent section, the utility of TMB as a clinically meaningful biomarker for PD1/CTLA-4 combination immunotherapy was demonstrated by primary results from CheckMate 227 part 1, which showed that the study met the coprimary end point of significantly longer PFS with nivolumab plus ipilimumab versus chemotherapy in patients with TMB $\geq 10$ mutations/Mb [47]. Although the Phase III MYSTIC study failed to show a significant OS advantage with first-line durvalumab plus tremelimumab versus chemotherapy in patients with $\geq 25 \%$ PD-L1 expression (coprimary end point), recent results of an exploratory analysis showed improved OS with the combination therapy versus chemotherapy in patients with a blood TMB $\geq 16$ mutations/Mb (HR: 0.62; 95\% CI: 0.45-0.86) [48].

\section{Mechanism of action}

Cancer cells have developed multiple strategies to evade immune surveillance by suppressing activation and effector functions of cells of the innate and adaptive immune system [49]. Immune checkpoint blockade has become an essential strategy in the fight against cancer as a means to restore anticancer immunity [12,50]. Currently approved checkpoint inhibitors in cancer treatment inhibit either the CD28/CTLA-4 system of immune modulation (ipilimumab) or the interaction between PD-1 and PD-L1 (atezolizumab, avelumab, durvalumab, nivolumab and pembrolizumab).

\section{CTLA-4 inhibition}

CTLA-4 is not expressed on naive and resting memory $T$ cells but highly expressed on activated $T$ cells, where it competes with CD28 for binding to CD80 and CD86 expressed on antigen-presenting cells [12,50]. Whereas CD28 engagement amplifies T-cell receptor (TCR) signaling as a costimulator, CTLA- 4 engagement reduces the 
amplitude of T-cell responses. Due to its higher affinity to CD80 and CD86 compared with CD28, CTLA-4 prevents sustained costimulation of TCR signaling after antigen engagement. This negative regulation of TCRmediated effector functions by CTLA- 4 is essential for the maintenance of immune tolerance. In cancers with high proportions of tumor antigen-reactive $\mathrm{T}$ cells, such as melanoma, CTLA- 4 inhibition is most likely to provide clinical benefit by restoring antitumor immunity [12].

\section{PD-1 inhibition}

PD-1 is expressed on activated T cells, and engagement by its ligands, PD-L1 and PD-L2, inhibits TCR-mediated effector functions [12]. Observations that PD-L1 is often expressed by tumor cells and PD-1 is highly expressed on tumor-infiltrating lymphocytes suggested that PD-1 engagement by PD-L1 provided a mechanism of immune evasion in the tumor microenvironment that might be reversible by blocking PD-1/PD-L1 interaction. Although tumor PD-L1 expression is in part intrinsic, it can also be the result of adaptive immune resistance mediated by inflammatory cytokines, particularly IFN- $\gamma$. In addition to tumor cells, PD-L1-expressing, tumor-infiltrating myeloid cells may induce T-cell inhibition and thus contribute to immune evasion [12].

\section{Synergy of dual checkpoint inhibition}

In cancer patients, CTLA- 4 appears to regulate immune responses early in T-cell activation, whereas PD- 1 inhibits the effector phase of T-cell activity in the tumor microenvironment [50]. CTLA-4 is mainly expressed on CD4 ${ }^{+}$ cells (helper T cells), and CTLA-4-mediated suppression of antitumor immunity most likely occurs at sites of T-cell activation in secondary lymphoid organs. In contrast, PD-1-mediated suppression of antitumor immunity relies primarily on the suppression of effector T-cell responses in the tumor microenvironment. Given the distinct but complementary mechanisms of actions of CTL4-A and PD-1 inhibition, dual checkpoint inhibition has the potential for synergistic antitumor activity by increasing the number of tumor-specific $T$ cells that remain activated to carry out effector functions. Consistent with this hypothesis, dual checkpoint inhibition in preclinical melanoma models resulted in the rejection of $65 \%$ of preimplanted tumors versus $10 \%$ with CTLA- 4 inhibition alone [51]. Furthermore, dual checkpoint inhibition greatly improved the ratios of effector $\mathrm{T}$ cells to regulatory $\mathrm{T}$ cells and myeloid-derived suppressor cells, suggesting an overall enhancement of antitumor immunity [51].

\section{Pharmacology}

\section{Nivolumab}

Nivolumab is a genetically engineered, fully human IgG4 monoclonal antibody against human PD-1 that binds to activated human $T$ cells with high affinity $\left(K_{D}=2.6 \mathrm{nM}\right)$ [52]. Nivolumab has been shown to inhibit the interaction of PD-1 with PD-L1 (concentration at half-maximal inhibition $\left[\mathrm{IC}_{50}\right]=2.52 \mathrm{nM}$ ) and PD-L2 $\left(\mathrm{IC}_{50}=2.59\right.$ nM) [53] and to enhance tumor antigen-specific T-cell proliferation and cytokine secretion in vitro [54].

Nivolumab has linear pharmacokinetics, with a terminal elimination half-life of approximately 25 days and doseindependent, time-varying clearance for doses of $0.1-20 \mathrm{mg} / \mathrm{kg}$ in patients with solid tumors [27,55]. Steady-state concentrations with multiple dosing at $3 \mathrm{mg} / \mathrm{kg}$ every 2 weeks (Q2W) are reached at 12 weeks, and exposure is dose proportional for doses of $0.1-10 \mathrm{mg} / \mathrm{kg} \mathrm{Q2W} \mathrm{[27].} \mathrm{A} \mathrm{model-based} \mathrm{population} \mathrm{pharmacokinetic} \mathrm{analysis} \mathrm{in} 1895$ patients from 11 clinical studies found no clinically relevant effects of renal impairment or hepatic impairment on nivolumab clearance [55]. PD-1 occupancy on circulating T cells after a single dose was independent of the dose. Mean peak occupancy 4-24 h after dosing was $85 \%$, and a sustained mean occupancy of more than $70 \%$ was observed at $\geq 2$ months after infusion, irrespective of dose [52].

\section{Ipilimumab}

Ipilimumab, a fully human IgG1 monoclonal antibody that prevents CTLA- 4 on activated T cells from binding to its ligands on antigen-presenting cells, has been shown to increase the percentages of circulating activated CD8 ${ }^{+}$ and $\mathrm{CD}^{+} \mathrm{T}$ cells and to enhance humoral immunity in patients with advanced melanoma [56]. Ipilimumab exhibited linear and time-invariant pharmacokinetics with dose-proportional exposure, which was not affected by moderate renal impairment or mild hepatic impairment [57,58]. The mean terminal half-life was 15 days [58]. Based on median steady-state trough concentrations, the probability of immune-related adverse events (AEs) grade $\geq 3$ was estimated to be 3,13 and $24 \%$ for doses of $0.3,3$ and $10 \mathrm{mg} / \mathrm{kg}$, respectively [56]. 


\section{Nivolumab plus ipilimumab}

Combined administration of nivolumab $3 \mathrm{mg} / \mathrm{kg}$ and ipilimumab $1 \mathrm{mg} / \mathrm{kg}$ did not change the clearance of nivolumab or ipilimumab [27]. A model-based analysis of the exposure-response relationship for different dose combinations of nivolumab and ipilimumab was conducted in 232 patients with previously untreated NSCLC [59]. The results suggested that adding a low dose of ipilimumab (1 mg/ $/ \mathrm{kg}$ Q6W or Q12W) to nivolumab $3 \mathrm{mg} / \mathrm{kg}$ Q2W was not associated with a significant increase in the risk of AEs leading to discontinuation or death. In contrast, the model predicted greater tumor shrinkage at week 12 with the combination regimens compared with nivolumab alone [59].

\section{Clinical efficacy of first-line nivolumab plus ipilimumab}

Rationale for first-line nivolumab plus ipilimumab in NSCLC

The rationale for evaluating dual-checkpoint inhibition for first-line therapy in NSCLC was based on mechanistic considerations as well as preclinical and clinical evidence. As discussed above, CTLA- 4 and PD-1 inhibition constitute distinct and complementary mechanisms to restore antitumor immunity. Furthermore, previous clinical experience in other cancers, particularly melanoma [60-62] and renal cell carcinoma [63,64], demonstrated that combination immunotherapy with nivolumab plus ipilimumab significantly improved outcomes compared with established treatments, while maintaining acceptable tolerability. Results from the Phase III CheckMate 067 study showed that in patients with previously untreated advanced melanoma, nivolumab plus ipilimumab was associated with significantly longer PFS and OS than ipilimumab alone. Outcomes with the combination were also improved compared with nivolumab monotherapy, although the differences in OS were not statistically significant [61,62]. Three-year OS rates with ipilimumab, nivolumab and combination therapy were 34,52 and 58\%, respectively [62]. In intermediate- and poor-risk patients with previously untreated advanced renal-cell carcinoma, OS was significantly longer and the objective response rate (ORR) significantly higher with nivolumab plus ipilimumab than sunitinib [64].

\section{PD-L1 \& TMB as predictors of response to immunotherapy}

PD-L1 expression has historically been regarded as an important biomarker of response to PD-1 and PD-L1 inhibitors, based on mechanism of action. In addition, clinical outcomes in NSCLC associated with PD-1/PD-L1 inhibitor therapy tend to improve with increasing PD-L1 expression [24,26,32,65,66]. However, both nivolumab and atezolizumab have shown improved outcomes compared with docetaxel in the second-line treatment of patients with NSCLC and less than 1\% PD-L1 expression, even though the absolute treatment benefit of PD-1/PD-L1 inhibition in this patient population was often reduced $[22,23,25]$. Results of a post hoc analysis from CheckMate 026 suggested that the use of TMB as a predictive biomarker instead of or in addition to PD-L1 expression may be effective in the selection of patients with advanced NSCLC who are likely to benefit from immunotherapy [38].

The rationale for using TMB as a biomarker for antitumor response is based on the concept that insertion and deletion mutations give rise to neoantigens, which in turn are determinants of tumor immunogenicity [67]. TMB has been identified as a predictor of response to immunotherapy across diverse cancers [68-70], including melanoma [71,72], urothelial carcinoma [73,74], small-cell lung cancer (SCLC) [75] and NSCLC [38,39,45,46]. Hellmann et al. retrospectively evaluated the relationship between TMB and efficacy of combination immunotherapy with nivolumab plus ipilimumab in both patients with SCLC and those with NSCLC. In patients with previously treated SCLC, median OS was 22 months for patients in the highest TMB tertile compared with less than 4 months for all other patients [75]. In patients with previously untreated NSCLC, TMB was a predictor of response and PFS, independent of PD-L1 expression [46].

A current limitation in comparing TMB-related outcomes across studies in NSCLC is the lack of a universally adopted standard for the definition and determination of TMB. Both whole-exome sequencing (WES) [38,39,46] and next-generation sequencing using specific gene panels $[47,48]$ have been used to determine TMB expressed as mutations per whole exome or mutations per megabase, respectively. In addition, the type of mutations included for the determination of TMB may vary between studies. Although it has been shown that TMB estimates by nextgeneration sequencing and WES may show good correlation [45], the heterogeneity in defining and determining TMB likely affects patient selection. Appropriate cut-offs for patient selection in clinical trials thus may need to be determined experimentally for each agent, including the use of receiver operating characteristic analyses in the targeted patient population. Currently, tumor biopsies are generally used to determine TMB; however, a blood-based assay was recently used in the MYSTIC study [48]. 
Phase I/II studies

CheckMate 012 (NCT01454102) was a multicohort Phase I study that evaluated the safety and clinical activity of nivolumab-based therapies, including nivolumab as monotherapy [65] and in combination with ipilimumab [76] or chemotherapy [77] in patients with previously untreated advanced NSCLC. Of six nivolumab-plus-ipilimumab combination regimens tested, four were excluded from further consideration for clinical development because of poor tolerability or insufficient clinical activity [76]. The two remaining regimens combined nivolumab $3 \mathrm{mg} / \mathrm{kg}$ Q2W, a dose demonstrated to be safe and effective for the treatment of advanced NSCLC [22,23], with a low dose of ipilimumab (1 mg/kg Q6W or Q12W). The ORR among all treated patients in these two cohorts $(\mathrm{n}=77)$ was $43 \%$ (Table 1). In contrast, the ORR in a cohort treated with nivolumab monotherapy was only $23 \%$, and ORRs in subgroups defined by PD-L1 expression were consistently lower with nivolumab than nivolumab plus low-dose ipilimumab [76]. In the two cohorts treated with nivolumab plus low-dose ipilimumab, patients with $\geq 1 \%$ PD-L1 expression had a higher ORR and improved PFS and OS compared with patients who had less than $1 \%$ PD-L1 expression (Table 1) [76,78]. Results of a post hoc analysis showed that ORR and PFS were more favorable in patients with TMB more than 158 mutations (the median value based on WES) than in those with lower TMB (Table 1) [46], and in a multivariable analysis including PD-L1 expression, tumor histology, smoking status, performance status and tumor burden, TMB was independently associated with ORR and PFS [46].

Results of the Phase II CheckMate 568 study confirmed that high TMB (determined by the FoundationOne CDx assay), irrespective of PD-L1 status, is associated with improved rates of response to nivolumab plus ipilimumab in patients with advanced NSCLC [79]. ORRs were 9, 15 and $44 \%$ for patients with $<5,5$ to $<10$ and $\geq 10$ mutations/Mb, respectively; however, no additional benefit was noted for patients with higher TMB, suggesting that a TMB of $\geq 10$ mutations/Mb is a clinically useful cut-off for the selection of patients most likely to respond to first-line nivolumab plus ipilimumab.

The study did not find a meaningful correlation between TMB and PD-L1 expression [79]. Although both PD-L1 expression $\geq 1 \%$ and $\mathrm{TMB} \geq 10$ mutations/Mb were associated with higher ORR, PD-L1 expression appeared to have little influence on ORR in patients with high TMB (Table 1). Patients with $\geq 10$ mutations/Mb $(\mathrm{n}=48)$ had an ORR of $47 \%$ if they had $\geq 1 \%$ PD-L1 expression compared with an ORR of $42 \%$ if they had less than $1 \%$ PD-L1 expression. In contrast, patients with less than ten mutations/Mb ( $\mathrm{n}=50)$ had an ORR of $18 \%$ if they had $\geq 1 \%$ PD-L1 expression compared with an ORR of 5\% if they had less than 1\% PD-L1 expression. Furthermore, in all patients treated with nivolumab plus ipilimumab $(n=288)$, the median PFS was almost three-times as long in those with $\geq 1 \%$ PD-L1 expression as in those with less than $1 \%$ PD-L1 expression (Table 1).

\section{Phase III study: CheckMate 227}

CheckMate 227 (NCT02477826) is an ongoing, large, multipart study evaluating the efficacy and safety of various nivolumab-based regimens, including nivolumab, nivolumab plus ipilimumab and nivolumab plus chemotherapy, in unselected, PD-L1-selected and TMB-selected patient populations with previously untreated advanced NSCLC [47]. A primary end point in part 1 of the study is OS with nivolumab plus ipilimumab versus chemotherapy in patients whose tumors express PD-L1, and results for this end point are pending. Based on the findings of CheckMate 568, PFS with nivolumab plus ipilimumab versus chemotherapy in patients with TMB $\geq 10$ mutations/Mb was chosen as an additional coprimary end point in part 1 of the study. This end point was added after the completion of enrollment but before the database lock. TMB was defined as the number of somatic, coding base substitutions and short insertions and deletions (indels) per Mb of genome examined, and was determined by the FoundationOne CDx assay [82-84]. Because the biopsy material collected at baseline was preferentially used for PD-L1 analysis, many patients had insufficient material for TMB analysis. Consequently, only 58\% of all randomized patients were evaluable for efficacy analyses by TMB. Nonetheless, baseline characteristics in these patients were balanced between treatment arms and similar to those of all randomized patients [47].

In CheckMate 227 part 1, a total of 1166 patients were randomly assigned to treatment with nivolumab $3 \mathrm{mg} / \mathrm{kg}$ Q2W plus ipilimumab $1 \mathrm{mg} / \mathrm{kg}$ Q6W, or histology-based platinum doublet chemotherapy for up to four 3-week cycles. Of those, 299 patients were evaluable for the coprimary end point of PFS in patients with TMB $\geq 10$ mutations/Mb. The study met its coprimary end point, showing a median PFS of 7.2 months with nivolumab plus ipilimumab versus 5.5 months with chemotherapy (HR: 0.58; 97.5\% CI: 0.41-0.81; p < 0.001; Table 1). Although the ORR was higher with nivolumab plus ipilimumab than chemotherapy (45 vs $27 \%$ ), the most striking difference between the two treatment arms was in the durability of responses. The 1-year PFS rate with nivolumab plus ipilimumab was more than three-times that with chemotherapy ( 43 vs 13\%; Table 1), and the percentage 
Table 1. Efficacy of nivolumab plus ipilimumab in CheckMate 012, 568 and 227.

CheckMate 012 (nivolumab 3 mg/kg Q2W + ipilimumab 1 mg/kg Q6W or Q12W)

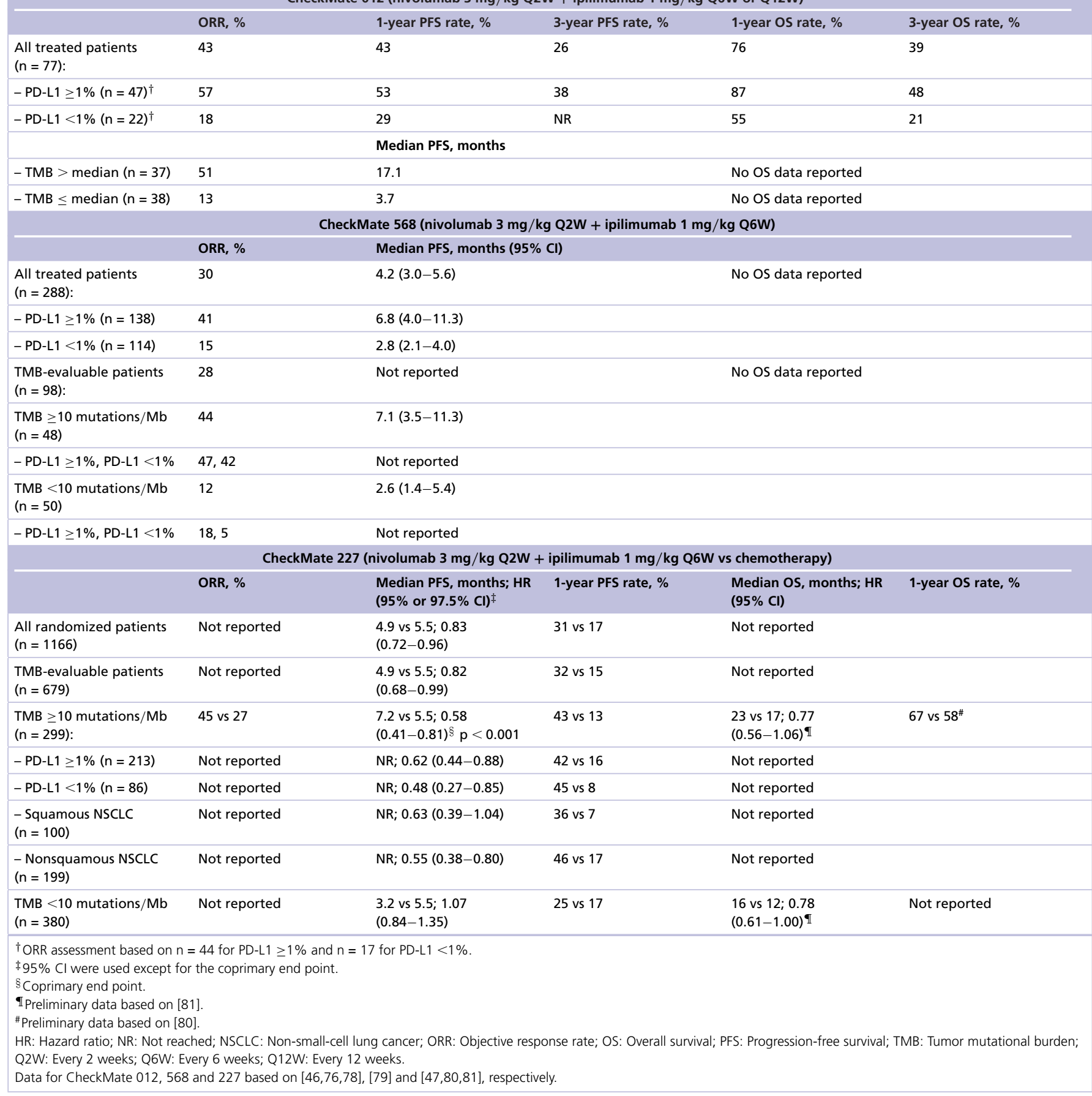

of patients with a complete or partial response who maintained this response for at least 1 year was $68 \%$ with nivolumab plus ipilimumab versus $25 \%$ with chemotherapy [47].

The PFS benefit of combination immunotherapy in patients with $\geq 10$ mutations/Mb was consistent across subgroups defined by age, gender, tumor histology or PD-L1 status. Importantly, outcomes were similar in patients with less than $1 \%$ and those with $\geq 1 \%$ tumor PD-L1 expression, with estimated 1-year PFS rates of 45 and $42 \%$, respectively (Table 1) [47]. Overall, patients with TMB $<10$ mutations/Mb did not gain a significant PFS advantage with combination immunotherapy versus chemotherapy (HR: 1.07; 95\% CI: 0.84-1.35); those treated with nivolumab plus ipilimumab had a median PFS of 3.2 months (vs 5.5 months with chemotherapy) and a 
1-year PFS rate of $25 \%$ (vs 17\% with chemotherapy) [47]. These data demonstrate that PFS with nivolumab plus ipilimumab was highly sensitive to patients' TMB, whereas PFS with chemotherapy was not affected by TMB.

CheckMate 227 part 1 included a treatment arm of nivolumab monotherapy in patients with $\geq 1 \%$ tumor PD-L1 expression. In patients with $\geq 1 \%$ tumor PD-L1 expression and TMB $\geq 10$ mutations/Mb $(n=203)$, the median PFS was longer with nivolumab plus ipilimumab (7.1 months) than nivolumab monotherapy (4.2 months; HR: 0.75; 95\% CI: 0.53-1.07). In addition, comparison of PFS with nivolumab monotherapy versus chemotherapy in patients with $\geq 1 \%$ PD-L1 expression and TMB $\geq 13$ mutations/Mb ( $\mathrm{n}=150)$, a secondary end point, showed no significant differences in PFS between the two treatments (HR: 0.95; 97.5\% CI: 0.61-1.48; $p=0.78$ ) [47].

Overall, the published data from CheckMate 227 part 1 demonstrate that combination immunotherapy with nivolumab plus ipilimumab provides a significant PFS advantage over chemotherapy in patients with TMB $\geq 10$ mutations/Mb, due to increased probability and durability of response. A corresponding descriptive analysis of OS based on a minimum follow-up of 14.2 months was presented at the 2018 Annual Meeting of the American Association for Cancer Research [80] and subsequently updated in a recent press release related to an ongoing regulatory review of the available study results (Table 1) [81]. Nivolumab plus ipilimumab was associated with a median OS of 23.0 versus 16.7 months with chemotherapy and an estimated $23 \%$ relative reduction in the risk of death (HR: 0.77; 95\% CI: 0.56-1.06). The press release also provided previously unpublished OS data from an exploratory analysis in patients with TMB $<10$ mutations/Mb. In this patient group, the median OS was 16.2 months with nivolumab plus ipilimumab versus 12.4 months with chemotherapy (HR: 0.78 ; $95 \%$ CI: 0.61-1.00) [81]. The study is continuing for the evaluation of the coprimary end point of OS with nivolumab plus ipilimumab versus chemotherapy in PD-L1-selected patients.

\section{Safety \& tolerability}

Nivolumab and ipilimumab largely lack the toxicities typically associated with chemotherapy, including hematologic toxicities and specific gastrointestinal disturbances such as nausea and vomiting. However, US package inserts for nivolumab and ipilimumab include warnings for a number of immune-related events, including pneumonitis, colitis (nivolumab only), hepatitis, endocrinopathies, nephritis, renal dysfunction, skin reactions (nivolumab only), infusion reactions and encephalitis [27,58]. Similar warnings and precautions are included in the corresponding product labeling by the EMA $[28,85]$.

Dose escalation with nivolumab at doses of $0.1-20 \mathrm{mg} / \mathrm{kg}$ Q2W in patients with solid tumors did not identify a maximum tolerated dose, and the rates of potentially immune-related AEs were similar across doses [86,87]. Furthermore, the nivolumab regimen of $3 \mathrm{mg} / \mathrm{kg}$ Q2W used in CheckMate 227 in combination with ipilimumab $1 \mathrm{mg} / \mathrm{kg}$ Q6W has been shown to be overall better tolerated by patients with advanced NSCLC than chemotherapy, due to consistently lower rates of any-grade and grade 3 or 4 treatment-related AEs (TRAEs) [22,23,38]. In contrast to nivolumab, ipilimumab has shown highly dose-dependent rates of immune-related AEs [56], and safety data from patients with advanced melanoma treated with nivolumab plus ipilimumab in CheckMate 067 indicated that ipilimumab at the high dose of $3 \mathrm{mg} / \mathrm{kg}$ Q3W dominated the AE profile of the combination [61]. However, a model-based analysis of safety data from CheckMate 012 suggested that the addition of ipilimumab at the low dose of $1 \mathrm{mg} / \mathrm{kg}$ Q6W to nivolumab $3 \mathrm{mg} / \mathrm{kg}$ Q2W would not have a significant impact on safety and tolerability in patients with previously untreated advanced NSCLC [59].

Table 2 summarizes the safety profile of nivolumab plus low-dose ipilimumab in previously untreated patients with NSCLC. In CheckMate 227, nivolumab plus low-dose ipilimumab was associated with similar rates of TRAEs as chemotherapy (75 vs $81 \%$ ) but higher rates of serious TRAEs ( 24 vs 14\%) and TRAEs leading to discontinuation (17 vs 9\%) [47]. The most common potentially immune-related TRAEs associated with nivolumab plus ipilimumab were skin reactions $(34 \%)$ and endocrine events $(23 \%)$, most of which were grade 1 or 2 . The most common grade 3 or 4 potentially immune-related TRAEs were hepatic events (8\%). Pneumonitis was the most common serious TRAE associated with nivolumab plus ipilimumab ( $4 \%$ of patients), the most common TRAE leading to discontinuation (3\% of patients), and the cause of three deaths in the nivolumab-plus-ipilimumab arm; four additional treatment-related deaths in this treatment arm were attributed to myocarditis, acute tubular necrosis, circulatory collapse and cardiac tamponade [47].

Analyses of patient-reported outcomes in CheckMate 227 found that patients with TMB $\geq 10$ mutations/Mb being treated with nivolumab plus ipilimumab experienced more rapid, durable and clinically meaningful improvements in disease-related symptom burden and health-related quality of life (HRQoL) than those being treated with chemotherapy, despite the potential for immune-related AEs [88,89]. Based on results obtained with the lung 
Table 2. Summary of treatment-related adverse events with nivolumab plus low-dose ipilimumab.

\begin{tabular}{|c|c|c|c|c|}
\hline \multirow[t]{2}{*}{ Percent of patients } & \multicolumn{2}{|c|}{ CheckMate $568^{\ddagger}(\mathrm{N}=288)$} & \multicolumn{2}{|c|}{ CheckMate $227^{\S}(\mathrm{N}=576)$} \\
\hline & Any grade & Grade 3 or 4 & Any grade & Grade 3 or 4 \\
\hline Any event & 80 & 29 & 75 & 31 \\
\hline Any serious event & Not reported & Not reported & 24 & 18 \\
\hline $\begin{array}{l}\text { Any event leading to } \\
\text { discontinuation }\end{array}$ & 16 & 9 & 17 & 12 \\
\hline \multicolumn{5}{|l|}{ Events in $\geq 10 \%$ of patients: } \\
\hline - Pruritus & 16 & 1 & 14 & $<1$ \\
\hline - Fatigue & 25 & 2 & 13 & 1 \\
\hline - Decreased appetite & 10 & $<1$ & 13 & $<1$ \\
\hline - Hypothyroidism & Not reported & Not reported & 12 & $<1$ \\
\hline - Asthenia & Not reported & Not reported & 10 & 1 \\
\hline - Skin & 30 & 3 & 34 & 4 \\
\hline - Endocrine & 19 & 2 & 23 & 4 \\
\hline - Gastrointestinal & 25 & 5 & 18 & 2 \\
\hline - Hepatic & 12 & 4 & 15 & 8 \\
\hline - Pulmonary & 7 & 2 & 8 & 3 \\
\hline - Renal & 1 & $<1$ & 4 & 1 \\
\hline $\begin{array}{l}\text { - Hypersensitivity/infusion } \\
\text { reaction }\end{array}$ & 7 & 1 & 4 & 0 \\
\hline $\begin{array}{l}\text { † Maculopapular rash in Check } \\
\text { ¥Based on reference [79]. } \\
\text { § Based on reference [47]. }\end{array}$ & 568. & & & \\
\hline
\end{tabular}

cancer symptom scale, patients treated with nivolumab plus ipilimumab on average achieved clinically meaningful improvements in disease-related symptoms, including fatigue and dyspnea. Patients treated with the combination immunotherapy on average also achieved the UK population norm for scores on the EuroQol three-level fivedimension HRQoL questionnaire visual analog scale and utility index (generic measures of HRQoL), suggesting a favorable HRQoL for surviving patients [88].

\section{Regulatory affairs}

At the time of writing this article, nivolumab plus ipilimumab is not approved for the first-line treatment of patients with advanced NSCLC and TMB $\geq 10$ mutations/Mb. A previous application for this indication to the FDA was withdrawn in January 2019 to await further evidence on the relationship between TMB and PD-L1, which requires final results from Checkmate 227 part 1 on the efficacy and safety of nivolumab plus ipilimumab versus chemotherapy in patients with $\geq 1 \%$ PD-L1 expression [90]. However, a corresponding application remains under review by the Committee for Medicinal Products for Human Use of the EMA [81]. In the USA, combination regimens of nivolumab plus ipilimumab are approved for the treatment of patients with unresectable or metastatic melanoma, patients with intermediate- or poor-risk, previously untreated advanced renal cell carcinoma, and patients 12 years and older with microsatellite instability-high or mismatch repair deficient metastatic colorectal cancer whose tumors have progressed following treatment with a fluoropyrimidine, oxaliplatin and irinotecan [27]. In the European Union, the combination of nivolumab and ipilimumab is approved for the treatment of advanced (unresectable or metastatic) melanoma in adults [28], and the treatment of patients with intermediate- or poor-risk, previously untreated advanced renal cell carcinoma [91]. 


\section{Conclusion}

The available results from CheckMate 227 demonstrate that dual checkpoint inhibition with nivolumab plus low-dose ipilimumab improves response and prolongs PFS compared with standard histology-based chemotherapy in patients with previously untreated advanced NSCLC and TMB $\geq 10$ mutations/Mb. Due to the hierarchical statistical analysis plan of CheckMate 227, the corresponding comparison of OS did not qualify for an inferential statistical analysis, and a statistically significant OS benefit with nivolumab plus ipilimumab versus chemotherapy has not been demonstrated. In this context, it is worth noting that the sample size for this comparison $(\mathrm{n}=299)$ was considerably smaller than corresponding sample sizes in other studies of first-line combination therapy [17-19]. However, the available OS data showed a trend toward improvement with nivolumab plus ipilimumab, with a large numerical difference in estimated median OS (23.0 vs 16.7 months), a finding that we believe is clinically meaningful. A similar trend in favor of nivolumab plus ipilimumab versus chemotherapy was observed in patients with $\mathrm{TMB}<10$ mutations/Mb, although median OS in both treatment arms (16.2 vs 12.4 months) was much shorter in this patient group than patients with $\mathrm{TMB} \geq 10$ mutations/Mb. Pending final OS results from CheckMate 227 part 1 may provide a better understanding of the relationship between TMB and PD-L1 expression and the combined impact of these two biomarkers on OS. The potential future role of first-line nivolumab plus ipilimumab in the management of advanced NSCLC will depend on the assessment of the clinical benefit observed across different patient populations, including those selected on the basis of TMB or PD-L1 expression.

\section{Author's contributions}

All the authors contributed to the content development and the writing of this Review and approved the final draft.

Financial \& competing interests disclosure

M Reck has served as a consultant for AbbVie, Amgen, AstraZeneca, Boehringer Ingelheim, Bristol-Myers Squibb, Celgene, Lilly, Merck, MSD, Novartis, Pfizer and Roche. H Borghaei has received clinical trial funding from Allergan (to his institution) and personal fees from AstraZeneca, Boehringer Ingelheim, Bristol-Myers Squibb, Celgene, Genentech, Genmab, Lilly, Merck, Novartis and Trovagen. KJ O'Byrne is an advisory board member with AstraZeneca, Boehringer Ingelheim, Bristol-Myers Squibb, Lilly Oncology, MSD, Novartis, Pfizer, Roche-Genentech and Teva; a speaker bureau member with AstraZeneca, Boehringer Ingelheim, BristolMyers Squibb, Lilly Oncology, MSD, Pfizer and Roche-Genentech; and has received travel grants for meetings from AstraZeneca, Boehringer Ingelheim, Bristol-Myers Squibb, MSD, Pfizer and Roche-Genentech. The authors have no other relevant affiliations or financial involvement with any organization or entity with a financial interest in or financial conflict with the subject matter or materials discussed in the manuscript apart from those disclosed.

Writing and editorial assistance was provided by Roland Tacke of Evidence Scientific Solutions, Inc., funded by Bristol-Myers Squibb.

\section{Open access}

This work is licensed under the Attribution-NonCommercial-NoDerivatives 4.0 Unported License. To view a copy of this license, visit http://creativecommons.org/licenses/by-nc-nd/4.0/ 
Executive summary

Immunotherapy in advanced non-small-cell lung cancer

- PD-1/PD-L1 inhibitor therapy has become the standard second-line treatment for patients with advanced non-small-cell lung cancer (NSCLC).

- Currently approved first-line immunotherapies include pembrolizumab monotherapy for patients with metastatic NSCLC and $\geq 50 \%$ PD-L1 expression, pembrolizumab in combination with platinum-based chemotherapy for patients with metastatic squamous or nonsquamous NSCLC, and atezolizumab in combination with bevacizumab, paclitaxel and carboplatin for patients with metastatic nonsquamous NSCLC.

Dual immune checkpoint inhibition with nivolumab plus ipilimumab

- To expand current treatment options, dual immune checkpoint inhibition with nivolumab plus ipilimumab as a chemotherapy-sparing first-line treatment strategy is being evaluated in the Phase III CheckMate 227 study.

- Nivolumab and ipilimumab have complementary mechanisms of action.

- Nivolumab plus ipilimumab has been shown to improve outcomes compared with established treatments in some advanced cancers.

Tumor mutational burden as predictive biomarker

- Tumor mutational burden (TMB) is an emerging biomarker of response to immunotherapy in multiple cancers, including response to nivolumab plus ipilimumab in advanced NSCLC.

- TMB and PD-L1 expression are independent biomarkers, and TMB can be used to identify patients with low PD-L1 expression who have an increased likelihood to respond to immunotherapy.

Efficacy of nivolumab plus ipilimumab in patients with untreated advanced NSCLC

- $\mathrm{TMB} \geq 10$ mutations/Mb was identified as a useful criterion for selecting patients most likely to derive progression-free survival benefit from combination immunotherapy with nivolumab plus ipilimumab.

- The Phase III CheckMate 227 study met its coprimary end point of superior progression-free survival with nivolumab $3 \mathrm{mg} / \mathrm{kg}$ Q2W plus ipilimumab $1 \mathrm{mg} / \mathrm{kg}$ Q6W versus chemotherapy in patients with TMB $\geq 10$ mutations $/ \mathrm{Mb}$.

- The treatment benefit of this combination was preserved in subgroups defined by tumor histology (squamous and nonsquamous) or PD-L1 expression ( $\geq 1$ and $<1 \%$ ).

Safety \& tolerability of nivolumab plus ipilimumab

- In CheckMate 227, nivolumab plus low-dose ipilimumab was associated with similar rates of treatment-related adverse events (TRAEs) as chemotherapy but higher rates of TRAEs leading to discontinuation (17 vs $9 \%$ ); pneumonitis was the most common TRAE leading to discontinuation ( $3 \%$ of patients).

- Patient-reported outcome analyses indicated that patients being treated with nivolumab plus ipilimumab on average experienced greater reductions in symptom burden and greater improvements in health-related quality of life than patients being treated with chemotherapy.

\section{Conclusion}

- Nivolumab plus ipilimumab has the potential to provide a chemotherapy-sparing first-line treatment option for patients with advanced NSCLC and TMB $\geq 10$ mutations/Mb, irrespective of tumor histology and PD-L1 expression.

- This combination could be particularly valuable in providing sustained disease control with improved health-related quality of life for patients who do not tolerate chemotherapy or wish to preserve chemotherapy as a future treatment option.

\section{References}

Papers of special note have been highlighted as: $\bullet$ of interest; $\bullet \bullet$ of considerable interest

1. Bray F, Ferlay J, Soerjomataram I, Siegel RL, Torre LA, Jemal A. Global cancer statistics 2018: GLOBOCAN estimates of incidence and mortality worldwide for 36 cancers in 185 countries. CA Cancer J. Clin. 68(6), 394-424 (2018).

2. Noone AM, Howler N, Krapcho M et al. SEER Cancer Statistics Review (CSR) 1975-2015. National Cancer Institute. https://seer.cancer.gov/csr/1975_2015/

3. Siegel RL, Miller KD, Jemal A. Cancer statistics, 2017. CA Cancer J. Clin. 67(1), 7-30 (2017).

4. American Cancer Society. What is non-small cell lung cancer? www.cancer.org/cancer/non-small-cell-lung-cancer/about/what-is-non-small-cell-lung-cancer.html

5. Samet JM, Avila-Tang E, Boffetta P et al. Lung cancer in never smokers: clinical epidemiology and environmental risk factors. Clin. Cancer Res. 15(18), 5626-5645 (2009).

6. Scagliotti GV, Parikh P, von Pawel J et al. Phase III study comparing cisplatin plus gemcitabine with cisplatin plus pemetrexed in chemotherapy-naive patients with advanced-stage non-small-cell lung cancer. J. Clin. Oncol. 26(21), 3543-3551 (2008).

7. Socinski MA, Bondarenko I, Karaseva NA et al. Weekly nab-paclitaxel in combination with carboplatin versus solvent-based paclitaxel plus carboplatin as first-line therapy in patients with advanced non-small-cell lung cancer: final results of a Phase III trial. J. Clin. Oncol. 30(17), 2055-2062 (2012). 
8. Patel JD, Socinski MA, Garon EB et al. PointBreak: a randomized Phase III study of pemetrexed plus carboplatin and bevacizumab followed by maintenance pemetrexed and bevacizumab versus paclitaxel plus carboplatin and bevacizumab followed by maintenance bevacizumab in patients with stage IIIB or IV nonsquamous non-small-cell lung cancer. J. Clin. Oncol. 31(34), 4349-4357 (2013).

9. Paz-Ares L, Mezger J, Ciuleanu TE et al. Necitumumab plus pemetrexed and cisplatin as first-line therapy in patients with stage IV non-squamous non-small-cell lung cancer (INSPIRE): an open-label, randomised, controlled Phase 3 study. Lancet Oncol. 16(3), 328-337 (2015).

10. Thatcher N, Hirsch FR, Luft AV et al. Necitumumab plus gemcitabine and cisplatin versus gemcitabine and cisplatin alone as first-line therapy in patients with stage IV squamous non-small-cell lung cancer (SQUIRE): an open-label, randomised, controlled Phase 3 trial. Lancet Oncol. 16(7), 763-774 (2015).

11. Zinner RG, Obasaju CK, Spigel DR et al. PRONOUNCE: randomized, open-label, Phase III study of first-line pemetrexed + carboplatin followed by maintenance pemetrexed versus paclitaxel + carboplatin + bevacizumab followed by maintenance bevacizumab in patients [w]ith advanced nonsquamous non-small-cell lung cancer. J. Thorac. Oncol. 10(1), 134-142 (2015).

12. Topalian SL, Drake CG, Pardoll DM. Immune checkpoint blockade: a common denominator approach to cancer therapy. Cancer Cell. 27(4), 450-461 (2015).

13. Ettinger DS, Wood DE, Aisner DL et al. Non-small cell lung cancer, version 2.2019, NCCN Clinical Practice Guidelines in Oncology. www.nccn.org/professionals/physician_gls/pdf/nscl.pdf

14. Planchard D, Popat S, Kerr K et al. Metastatic non-small cell lung cancer: ESMO Clinical Practice Guidelines for diagnosis, treatment and follow-up. Ann. Oncol. 29(Suppl. 4), iv192-iv237 (2018).

15. KEYTRUDA ${ }^{\circledR}$ (pembrolizumab) [package insert]. Merck \& Co. Inc., Whitehouse Station, NJ, USA, November 2018. www.merck.com/product/usa/pi_circulars/k/keytruda/keytruda_pi.pdf

16. Keytruda: EPAR - Product Information. (2018). www.ema.europa.eu/documents/product-information/keytruda-epar-product-information_en.pdf

17. Gandhi L, Rodríguez-Abreu D, Gadgeel S et al. Pembrolizumab plus chemotherapy in metastatic non-small-cell lung cancer. $N$. Engl. J. Med. 378(22), 2078-2092 (2018)

18. Paz-Ares L, Luft A, Vicente D et al. Pembrolizumab plus chemotherapy for squamous non-small-cell lung cancer. N. Engl. J. Med. 379(21), 2040-2051 (2018).

19. Socinski MA, Jotte RM, Cappuzzo F et al. Atezolizumab for first-line treatment of metastatic nonsquamous NSCLC. N. Engl. J. Med. 378(24), 2288-2301 (2018).

20. TECENTRIQ ${ }^{\circledR}$ (atezolizumab) [package insert]. Genentech, Inc., South San Francisco, CA, USA, December 2018. www.gene.com/download/pdf/tecentriq_prescribing.pdf

21. Pakkala S, Ramalingam SS. Personalized therapy for lung cancer: striking a moving target. JCI Insight. 3(15), 120858 (2018).

22. Brahmer J, Reckamp KL, Baas $\mathrm{P}$ et al. Nivolumab versus docetaxel in advanced squamous-cell non-small-cell lung cancer. $N$. Engl. J. Med. 373(2), 123-135 (2015).

-• Together, with Ref. 23, it established PD-1 inhibitor therapy as the new standard of treatment for patients with previously treated advanced non-small-cell lung cancer (NSCLC).

23. Borghaei H, Paz-Ares L, Horn L et al. Nivolumab versus docetaxel in advanced nonsquamous non-small-cell lung cancer. N. Engl. J. Med. 373(17), 1627-1639 (2015).

- Together, with Ref. 22, it established PD-1 inhibitor therapy as the new standard of treatment for patients with previously treated advanced NSCLC.

24. Herbst RS, Baas P, Kim DW et al. Pembrolizumab versus docetaxel for previously treated, PD-L1-positive, advanced non-small-cell lung cancer (KEYNOTE-010): a randomised controlled trial. Lancet 387(10027), 1540-1550 (2016).

25. Rittmeyer A, Barlesi F, Waterkamp D et al. Atezolizumab versus docetaxel in patients with previously treated non-small-cell lung cancer (OAK): a Phase 3, open-label, multicentre randomised controlled trial. Lancet 389(10066), 255-265 (2017).

26. Garon EB, Rizvi NA, Hui R et al. Pembrolizumab for the treatment of non-small-cell lung cancer. N. Engl. J. Med. 372(21), 2018-2028 (2015).

27. OPDIVO $^{\circledR}$ (nivolumab) [package insert]. Bristol-Myers Squibb Company, Princeton, NJ, USA, November 2018. https://packageinserts.bms.com/pi/pi_opdivo.pdf

28. Opdivo: EPAR - Product Information. (2018). www.ema.europa.eu/documents/product-information/opdivo-epar-product-information_en.pdf

29. Tecentriq: EPAR - Product Information. (2018). www.ema.europa.eu/documents/product-information/tecentriq-epar-product-information_en.pdf

30. Fehrenbacher L, von Pawel J, Park K et al. Updated efficacy analysis including secondary population results for OAK: a randomized Phase III study of atezolizumab versus docetaxel in patients with previously treated advanced non-small cell lung cancer. J. Thorac. Oncol. 13(8), 1156-1170 (2018). 
31. Herbst RS, Garon EB, Kim D et al. Long-term survival in patients (pts) with advanced NSCLC in the KEYNOTE-010 study overall and in pts who completed 2 years of pembrolizumab (pembro). Presented at: ESMO 2018 Congress. Munich, Germany, 19-23 October 2018.

32. Horn L, Spigel DR, Vokes EE et al. Nivolumab versus docetaxel in previously treated patients with advanced non-small-cell lung cancer: two-year outcomes from two randomized, open-label, Phase III trials (CheckMate 017 and CheckMate 057). J. Clin. Oncol. 35(35), 3924-3933 (2017).

33. Vokes EE, Ready N, Felip E et al. Nivolumab versus docetaxel in previously treated advanced non-small-cell lung cancer (CheckMate 017 and CheckMate 057): 3-year update and outcomes in patients with liver metastases. Ann. Oncol. 29(4), 959-965 (2018).

34. Gettinger S, Horn L, Jackman D et al. Five-Year follow-up of nivolumab in previously treated advanced non-small-cell lung cancer: results from the CA209-003 study. J. Clin. Oncol. 36(17), 1675-1684 (2018).

35. Reck M, Rodríguez-Abreu D, Robinson AG et al. Pembrolizumab versus chemotherapy for PD-L1-positive non-small-cell lung cancer. N. Engl. J. Med. 375(19), 1823-1833 (2016).

-• This landmark study demonstrated the value of PD-L1 expression as a biomarker for PD-1 inhibitor therapy in patients with untreated metastatic NSCLC and led to the first and so far only approval of single-agent immunotherapy in this patient population.

36. Brahmer J, Rodríguez-Abreu D, Robinson A et al. Updated analysis of KEYNOTE-024: pembrolizumab vs platinum-based chemotherapy for advanced NSCLC with PD-L1 TPS $\geq 50 \%$. J. Thorac. Oncol. 12(11 Suppl. 2), S1793-S1794, Abstract OA 1717.1706 (2017).

37. Lopes G, Wu Y-L, Kudaba I et al. Pembrolizumab (pembro) versus platinum-based chemotherapy (chemo) as first-line therapy for advanced/metastatic NSCLC with a PD-L1 tumor proportion score (TPS) $\geq 1 \%$ open-label, Phase 3 KEYNOTE-042 study. Presented at: American Society of Clinical Oncology Annual Meeting. Chicago, IL, USA, 1-5 June 2018.

38. Carbone DP, Reck M, Paz-Ares L et al. First-line nivolumab in stage IV or recurrent non-small-cell lung cancer. N. Engl. J. Med. 376(25), 2415-2426 (2017).

- Although the study did not meet its primary end point, its findings suggested limitations for PD-L1 expression as a predictor of response to first-line immunotherapy in advanced NSCLC and provided evidence supporting a role for tumor mutational burden (TMB) as an alternative biomarker of efficacy.

39. Rizvi NA, Hellmann MD, Snyder A et al. Mutational landscape determines sensitivity to PD-1 blockade in non-small cell lung cancer. Science 348(6230), 124-128 (2015).

- This detailed analysis provided strong support for the use of TMB as a predictive biomarker of response to PD-1 blockade in NSCLC.

40. Source: Roche. European Commission approves Roche's Tecentriq in combination with Avastin and chemotherapy for the initial treatment of people with a specific type of metastatic lung cancer. Press release: www.roche.com/media/releases/med-cor-2019-03-08.htm

41. Cappuzzo F, McCleod M, Hussein M et al. IMpower130: progression-free survival (PFS) and safety analysis from a randomised Phase 3 study of carboplatin + nab-paclitaxel $(\mathrm{CnP})$ with or without atezolizumab (atezo) as first-line (1L) therapy in advanced non-squamous NSCLC. Presented at: ESMO 2018 Congress. Munich, Germany, 19-23 October 2018.

42. Barlesi F, Nishio M, Cobo M et al. IMpower132: efficacy of atezolizumab (atezo) + carboplatin (carbo)/cisplatin (cis) + pemetrexed (pem) as 1L treatment in key subgroups with stage IV non-squamous non-small cell lung cancer (NSCLC). Presented at: ESMO 2018 Congress. Munich, Germany, 19-23 October 2018.

43. Socinski MA, Rittmeyer A, Shapovalov D et al. IMpower131: progression-free survival (PFS) and overall survival (OS) analysis of a randomised Phase III study of atezolizumab + carboplatin + paclitaxel or nab-paclitaxel vs carboplatin + nab-paclitaxel in $1 \mathrm{~L}$ advanced squamous NSCLC. Presented at: ESMO 2018 Congress. Munich, Germany, 19-23 October 2018.

44. Borghaei H, Hellmann MD, Paz-Ares L et al. Nivolumab + ipilimumab, nivolumab + chemotherapy, and chemotherapy in chemo-naive patients with advanced non-small cell lung cancer and $<1 \%$ tumor PD-L1 expression: results from CheckMate 227. Abstract 9001. Presented at: American Society of Clinical Oncology Annual Meeting. Chicago, IL, USA, 1-5 June 2018.

- In patients with previously untreated advanced NSCLC with less than 1\% PD-L1 expression, nivolumab plus chemotherapy was associated with a higher objective response rate, longer duration of response and significantly longer progression-free survival.

45. Rizvi H, Sanchez-Vega F, La K et al. Molecular determinants of response to anti-programmed cell death (PD)-1 and anti-programmed death-ligand 1 (PD-L1) blockade in patients with non-small-cell lung cancer profiled with targeted next-generation sequencing. J. Clin. Oncol. 36(7), 633-641 (2018).

-• It demonstrated the feasibility of using next-generation sequencing to accurately measure TMB and provided evidence that TMB and PD-L1 expression are independent predictors of response to PD-1 or PD-L1 inhibition in NSCLC.

46. Hellmann MD, Nathanson T, Rizvi $\mathrm{H}$ et al. Genomic features of response to combination immunotherapy in patients with advanced non-small-cell lung cancer. Cancer Cell. 33(5), 843-852.e4 (2018).

- It demonstrated the utility of TMB as an independent predictive biomarker of efficacy of nivolumab plus ipilimumab in patients with NSCLC. 
47. Hellmann MD, Ciuleanu TE, Pluzanski A et al. Nivolumab plus ipilimumab in lung cancer with a high tumor mutational burden. $N$. Engl. J. Med. 378(22), 2093-2104 (2018).

-• This is the first and so far only Phase III study to demonstrate significant clinical benefit of combination immunotherapy versus chemotherapy in patients with untreated advanced NSCLC by using TMB as a predictive biomarker.

48. Rizvi NA, Chul Cho B, Reinmuth $\mathrm{N}$ et al. Durvalumab with or without tremelimumab vs platinum-based chemotherapy as first-line treatment for metastatic non-small cell lung cancer: MYSTIC. Presented at: ESMO Immuno-Oncology Congress 2018. Geneva, Switzerland, 13-16 December 2018.

49. Vinay DS, Ryan EP, Pawelec G et al. Immune evasion in cancer: mechanistic basis and therapeutic strategies. Semin. Cancer Biol. 35(Suppl.), S185-S198 (2015).

50. Postow MA, Callahan MK, Wolchok JD. Immune checkpoint blockade in cancer therapy. J. Clin. Oncol. 33(17), 1974-1982 (2015).

51. Curran MA, Montalvo W, Yagita H, Allison JP. PD-1 and CTLA-4 combination blockade expands infiltrating T cells and reduces regulatory T and myeloid cells within B16 melanoma tumors. Proc. Natl Acad. Sci. USA 107(9), 4275-4280 (2010).

52. Brahmer JR, Drake CG, Wollner I et al. Phase I study of single-agent anti-programmed death-1 (MDX-1106) in refractory solid tumors: safety, clinical activity, pharmacodynamics, and immunologic correlates. J. Clin. Oncol. 28(19), 3167-3175 (2010).

53. Wang C, Thudium KB, Han M et al. In vitro characterization of the anti-PD-1 antibody nivolumab, BMS-936558, and in vivo toxicology in non-human primates. Cancer Immunol. Res. 2(9), 846-856 (2014).

54. Wong RM, Scotland RR, Lau RL et al. Programmed death-1 blockade enhances expansion and functional capacity of human melanoma antigen-specific CTLs. Int. Immunol. 19(10), 1223-1234 (2007).

55. Bajaj G, Wang X, Agrawal S, Gupta M, Roy A, Feng Y. Model-based population pharmacokinetic analysis of nivolumab in patients with solid tumors. CPT Pharmacometrics Syst. Pharmacol. 6(1), 58-66 (2017).

56. Feng Y, Roy A, Masson E, Chen TT, Humphrey R, Weber JS. Exposure-response relationships of the efficacy and safety of ipilimumab in patients with advanced melanoma. Clin. Cancer Res. 19(14), 3977-3986 (2013).

57. Feng Y, Masson E, Dai D, Parker SM, Berman D, Roy A. Model-based clinical pharmacology profiling of ipilimumab in patients with advanced melanoma. Br. J. Clin. Pharmacol. 78(1), 106-117 (2014).

58. YERVOY $^{\circledR}$ (ipilimumab) [package insert]. Bristol-Myers Squibb Company, Princeton, NJ, USA, July 2018. https://packageinserts.bms.com/pi/pi_yervoy.pdf

59. Zhao X, Feng Y, Wang X et al. Tumor growth dynamic modeling and safety analysis of nivolumab plus ipilimumab in first-line patients with non-small cell lung cancer. Presented at: American Society for Clinical Pharmacology and Therapeutics Annual Meeting. Washington, DC, USA, 15-18 March 2017.

60. Postow MA, Chesney J, Pavlick AC et al. Nivolumab and ipilimumab versus ipilimumab in untreated melanoma. N. Engl. J. Med. 372(21), 2006-2017 (2015).

61. Larkin J, Chiarion-Sileni V, Gonzalez R et al. Combined nivolumab and ipilimumab or monotherapy in untreated melanoma. $N$. Engl. J. Med. 373(1), 23-34 (2015).

62. Wolchok JD, Chiarion-Sileni V, Gonzalez R et al. Overall survival with combined nivolumab and ipilimumab in advanced melanoma. N. Engl. J. Med. 377(14), 1345-1356 (2017).

- Long-term follow-up data of this Phase III study showed that combination therapy with nivolumab plus ipilimumab or nivolumab monotherapy resulted in significantly longer survival of patients with advanced melanoma than monotherapy with ipilimumab alone.

63. Hammers HJ, Plimack ER, Infante JR et al. Safety and efficacy of nivolumab in combination with ipilimumab in metastatic renal cell carcinoma: the CheckMate 016 study. J. Clin. Oncol. 35(34), 3851-3858 (2017).

64. Motzer RJ, Tannir NM, McDermott DF et al. Nivolumab plus ipilimumab versus sunitinib in advanced renal-cell carcinoma. N. Engl. J. Med. 378(14), 1277-1290 (2018).

65. Gettinger S, Rizvi NA, Chow LQ et al. Nivolumab monotherapy for first-line treatment of advanced non-small-cell lung cancer. J. Clin Oncol. 34(25), 2980-2987 (2016).

66. Fehrenbacher L, Spira A, Ballinger M et al. Atezolizumab versus docetaxel for patients with previously treated non-small-cell lung cancer (POPLAR): a multicentre, open-label, Phase 2 randomised controlled trial. Lancet 387(10030), 1837-1846 (2016).

67. Turajlic S, Litchfield $\mathrm{K}, \mathrm{Xu} \mathrm{H}$ et al. Insertion-and-deletion-derived tumour-specific neoantigens and the immunogenic phenotype: a pan-cancer analysis. Lancet Oncol. 18(8), 1009-1021 (2017).

68. Goodman AM, Kato S, Bazhenova $\mathrm{L}$ et al. Tumor mutational burden as an independent predictor of response to immunotherapy in diverse cancers. Mol. Cancer Ther. 16(11), 2598-2608 (2017).

69. Yarchoan M, Hopkins A, Jaffee EM. Tumor mutational burden and response rate to PD-1 inhibition. N. Engl. J. Med. 377(25), 2500-2501 (2017).

70. Le DT, Durham JN, Smith KN et al. Mismatch repair deficiency predicts response of solid tumors to PD-1 blockade. Science 357(6349), 409-413 (2017). 
71. Snyder A, Makarov V, Merghoub T et al. Genetic basis for clinical response to CTLA-4 blockade in melanoma. N. Engl. J. Med. 371(23), 2189-2199 (2014).

72. Van Allen EM, Miao D, Schilling B et al. Genomic correlates of response to CTLA-4 blockade in metastatic melanoma. Science 350(6257), 207-211 (2015).

73. Rosenberg JE, Hoffman-Censits J, Powles $\mathrm{T}$ et al. Atezolizumab in patients with locally advanced and metastatic urothelial carcinoma who have progressed following treatment with platinum-based chemotherapy: a single-arm, multicentre, Phase 2 trial. Lancet 387(10031), 1909-1920 (2016).

74. Powles T, Loriot Y, Ravaud A et al. Atezolizumab vs chemotherapy in platinum-treated locally advanced or metastatic urothelial carcinoma: immune biomarkers, tumor mutational burden and clinical outcomes from the Phase III IMvigor211 study. Presented at: 2018 Genitourinary Cancers Symposium. San Francisco, CA, USA, 8-10 February 2018.

75. Hellmann MD, Callahan MK, Awad MM et al. Tumor mutational burden and efficacy of nivolumab monotherapy and in combination with ipilimumab in small-cell lung cancer. Cancer Cell. 33(5), 853-861.e4 (2018).

- Demonstrated the utility of TMB as an independent predictive biomarker of efficacy of nivolumab plus ipilimumab in patients with small-cell lung cancer.

76. Hellmann MD, Rizvi NA, Goldman JW et al. Nivolumab plus ipilimumab as first-line treatment for advanced non-small-cell lung cancer (CheckMate 012): results of an open-label, Phase 1, multicohort study. Lancet Oncol. 18(1), 31-41 (2017).

77. Rizvi NA, Hellmann MD, Brahmer JR et al. Nivolumab in combination with platinum-based doublet chemotherapy for first-line treatment of advanced non-small-cell lung cancer. J. Clin. Oncol. 34(25), 2969-2979 (2016).

78. Antonia SJ, Gettinger SN, Borghaei H et al. Nivolumab plus ipilimumab in advanced NSCLC: 3-year follow-up from CheckMate 012. Presented at: 19th World Conference on Lung Cancer. Toronto, Canada, 23-26 September 2018.

79. Ready N, Hellmann MD, Awad MM et al. First-line nivolumab plus ipilimumab in advanced non-small-cell lung cancer (CheckMate 568): outcomes by programmed death ligand 1 and tumor mutational burden as biomarkers. J. Clin. Oncol. 37(12), 992-1000 (2019).

80. Hellmann MD, Ciuleanu T-E, Pluzanski A et al. Nivolumab + ipilimumab vs platinum-doublet chemotherapy as first-line treatment for advanced non-small cell lung cancer: initial results from CheckMate 227. Presented at: American Association for Cancer Research 2018 Annual Meeting. Chicago, IL, USA, 14-18 April 2018.

81. Bristol-Myers Squibb Company. Bristol-Myers Squibb provides update on the ongoing regulatory review of Opdivo plus low-dose yervoy in first-line lung cancer patients with tumor mutational burden $\geq 10$ mut/Mb. Press release: https://news.bms.com/press-release/corporatefinancial-news/bristol-myers-squibb-provides-update-ongoing-regulatory-review

82. FoundationOne $\mathrm{CDx}^{\top \mathrm{M}}$. www.foundationmedicine.com/genomic-testing/foundation-one-cdx

83. Chalmers ZR, Connelly CF, Fabrizio D et al. Analysis of 100,000 human cancer genomes reveals the landscape of tumor mutational burden. Genome Med. 9(1), 34 (2017).

84. Sun JX, He Y, Sanford E et al. A computational approach to distinguish somatic vs. germline origin of genomic alterations from deep sequencing of cancer specimens without a matched normal. PLoS Comput. Biol. 14(2), e1005965 (2018).

85. Yervoy: EPAR - Product Information. (2018). www.ema.europa.eu/documents/product-information/yervoy-epar-product-information_en.pdf

86. Topalian SL, Hodi FS, Brahmer JR et al. Safety, activity, and immune correlates of anti-PD-1 antibody in cancer. N. Engl. J. Med. 366(26), 2443-2454 (2012).

87. Yamamoto N, Nokihara H, Yamada Y et al. Phase I study of nivolumab, an anti-PD-1 antibody, in patients with malignant solid tumors. Invest New Drugs 35(2), 207-216 (2017).

88. Brahmer J, Schenker M, Lee KH et al. CheckMate 227: patient-reported outcomes of first-line nivolumab + ipilimumab in high tumor mutational burden advanced NSCLC. Abstract OA05.06. Presented at: IASLC 19th World Conference on Lung Cancer. Toronto, Canada, 23-26 September 2018.

89. Reck M, Hellmann MD, Paz-Ares $\mathrm{L}$ et al. Nivolumab+ipilimumab vs platinum-doublet chemotherapy as first-line treatment for advanced non-small cell lung cancer: safety analysis and patient-reported outcomes from CheckMate 227. Abstract 9020. Presented at: American Society of Clinical Oncology Annual Meeting. Chicago, IL, USA, 1-5 June 2018.

90. Source: Bristol-Myers Squibb Company. Bristol-Myers Squibb reports fourth quarter and full year financial results. Press release: https://news.bms.com/press-release/corporatefinancial-news/bristol-myers-squibb-reports-fourth-quarter-and-full-year-fi-0

91. Source: Bristol-Myers Squibb Company. European Commission approves Opdivo (nivolumab) plus low-dose Yervoy (ipilimumab) for first-line treatment of patients with intermediate- and poor-risk advanced renal cell carcinoma. Press release:

https://news.bms.com/press-release/corporatefinancial-news/european-commission-approves-opdivo-nivolumab-plus-low-dose-ye 